\title{
A Case Study of Object and Occlusion Management on the eLabBench, a Mixed Physical/Digital Tabletop.
}

\author{
Aurélien Tabard ${ }^{1,2,3}$ \\ ${ }^{1}$ Université Lyon 1 \& CNRS \\ aurelien@tabard.fr
}

\author{
Simon Gurn ${ }^{2}$, Andreas Butz ${ }^{2}$ \\ ${ }^{2}$ University of Munich (LMU) \\ butz@ifi.lmu.de
}

\author{
Jakob E. Bardram ${ }^{3}$ \\ ${ }^{3}$ IT University of Copenhagen \\ bardram@itu.dk
}

\begin{abstract}
We investigate how users managed physical and digital objects during the longitudinal field deployment of a tabletop in a biology laboratory. Based on the analysis of 15 hours of video $\operatorname{logs}$, we detail the objects used, their presence, use and organization, in this particular setting. We propose to consider occlusion as a situation which should be prevented rather than reacted to, particularly to avoid distracting changes or animations. This implies (1) pre-positioning digital content in locations where it is not likely to be occluded and (2) acknowledging that some physical objects are deliberately put in occluding positions. Since users want to interact with such objects conveniently, occlusion management actions should not necessarily be triggered immediately.
\end{abstract}

\section{Author Keywords}

Tabletop; occlusion; object management; laboratory bench; tangible interaction.

\section{ACM Classification Keywords}

H.5.m. Information Interfaces and Presentation (e.g. HCI): Miscellaneous

\section{General Terms}

Human Factors; Design; Measurement.

\section{OBJECT MANAGEMENT ON TABLETOPS}

Interactive tabletops allow for simultaneous interaction with both physical and digital objects on their surface. As a consequence, occlusion of parts of the tabletop display by physical objects appears to be common. Over the past years, a set of techniques have been proposed to mitigate occlusion problems on tabletops. For instance, instead of displaying a full screen application, Cotting and Gross [3] proposed to display every window in independent optically deformed bubbles avoiding physical objects placed on the tabletop, while Brandl et al. proposed flexible menus to avoid objects [2]. An alternative approach explored by Javed et al. [6] and Khalilbeigi et al. [7] is to display information about the occluded digital content with different degree of details. For example, Javed et al. [6] proposed three stages: (i) awareness (knowledge that a particular target exists), (ii) identification (target is recognizable but cannot be interacted with), and (iii) access (the ability to fully interact with the target).

Permission to make digital or hard copies of all or part of this work for personal or classroom use is granted without fee provided that copies are not made or distributed for profit or commercial advantage and that copies bear this notice and the full citation on the first page. Copyrights for components of this work owned by others than ACM must be honored. Abstracting with credit is permitted. To copy otherwise, or republish, to post on servers or to redistribute to lists, requires prior specific permission and/or a fee. Request permissions from permissions@ acm.org.

ITS'13, October 6-9, 2013, St. Andrews, United Kingdom.

Copyright is held by the owner/author(s). Publication rights licensed to ACM. ACM 978-1-4503-2271-3/13/10...\$15.00.

http://dx.doi.org/10.1145/2512349.2512794

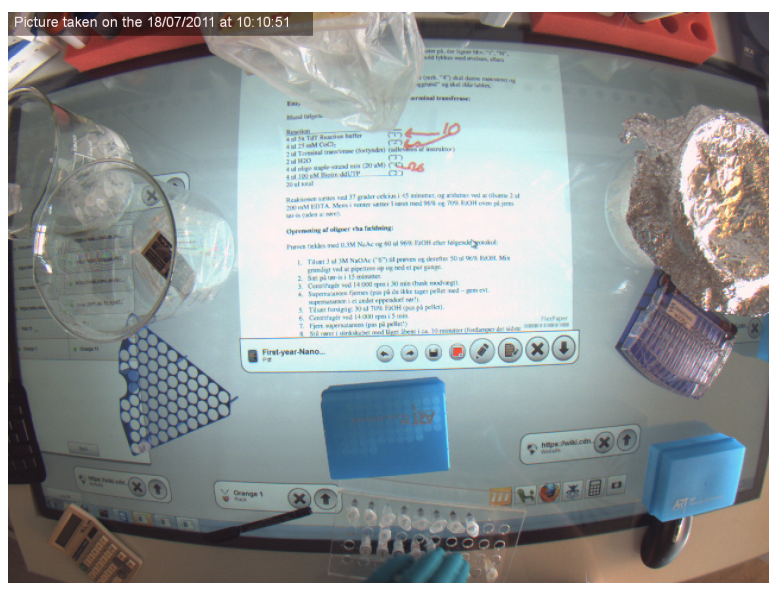

Figure 1. Top view of eLabBench, as recorded for data collection.

In order to design relevant and efficient occlusion management techniques, designers need an understanding of both objects and occlusion management on tabletops. Toney and Thomas study of reach [12], plus Scott et al. [8] work on territoriality in tabletop collaboration, and Steimle et al. [9] studies of grouping and sorting physical and digital paper-sized documents, led to the identifications of different functional areas of tabletops, for storage, manipulation and exchange, with specifics for digital and physical documents. However these studies were conducted in laboratories, with a limited set of physical items and predefined tasks.

We present an analysis of the placement and occlusion of objects on the eLabBench [11], a single-user tabletop for laboratory environments (shown in fig. 1). The analysis is based on video logs from the longitudinal deployment of the eLabBench. We describe how participants used and organized objects and analyze how they managed occlusion during their daily use in which they were free to pursue any task and use any object.

\section{DATA COLLECTION AND ANALYSIS}

Data was collected during the 16-week deployment of the eLabBench in 2011 [10]. The eLabBench is an interactive tabletop-based laboratory bench for synthetic biologists. It was designed to be used by one person at a time sitting on one side of the bench and facing a wall.

Participants were asked to use the eLabBench while executing an experimental protocol. They did not receive any specific directions, and used laboratory equipment as well as paper documents, pens and calculators. We analyzed video logs from 4 participants covering 15 hours of bench work, spanning 5 sessions lasting from 30 minutes to more than 8 hours. 
All participants were right-handed. We removed long periods of inactivity (e.g., lunch breaks or meetings), to focus on active periods at the bench. The videos were recorded with a Firefly camera mounted above the bench, taking 1280 x960 px pictures every 10 seconds (as shown in fig. 1). We are thus not discussing micro-interactions $(<10 s)$ or hand occlusion [13] in this study.

We coded the videos using Advene [1] by making spatial annotations of the objects put on the tabletop. The annotations of each object on the surface included (i) identity (by giving each object a name), (ii) time on surface, (iii) location on surface, and (iv) "type", either hybrid (i.e. tangibles which were tracked and had digital data attached to them, namely racks of tests tubes augmented with digital information visible to their side) or passive (i.e. objects neither tracked nor augmented like notebooks, pens, calculators or pipettes). We used color to code the type of the objects.

The coded and synthesized data is attached to the article. The additional data also contains explanations on how we computed occlusion levels and generated the heatmaps below.

\section{OBSERVATIONS}

Based on our observations of the eLabBench usage, we identified three main questions related to object management on tabletops; (i) What are the objects used? (ii) How are they organized on the tabletop surface? and (iii) How did the objects cause occlusion and how did the participants deal with it?

\section{Object Usage}

We present here a quantitative overview of the objects used over the 5 sessions: their quantity, size and presence.

\section{Quantity of objects}

We counted 89 physical objects used on the table (12 hybrid, 77 passive), with an average of 17.8 per session $(S D=6.3)$. We did not observe a significant correlation between the duration of a session and the number of objects used, for instance session 4 only lasted 32 minutes but involved almost as many objects as session 2 which was 15 times longer (see Table 1).

\begin{tabular}{|l|l|l|}
\hline Session & Objects used (Passive/Hybrid) & Time (min) \\
\hline 1 & $10(8 / 2)$ & 146 \\
\hline 2 & $28(27 / 1)$ & 508 \\
\hline 3 & $16(16 / 0)$ & 101 \\
\hline 4 & $24(18 / 3)$ & 32 \\
\hline 5 & $13(10 / 3)$ & 86 \\
\hline
\end{tabular}

Table 1. Overview of the objects used among the different sessions.

\section{Size of objects}

We identified four types of objects in respect of their size ${ }^{1,2}$ : (1) 34 small objects generating marginal occlusion (their size ranges from $3.5 \mathrm{~cm}^{2}$ and $8.8 \mathrm{~cm}^{2}$ ), composed of pens, pipettes, caps or small tubes. (2) 29 medium sized objects (between $9.5 \mathrm{~cm}^{2}$ and $13.7 \mathrm{~cm}^{2}$ ), composed of small boxes and racks, gloves and glass ware. (3) 23 large objects (between $14.1 \mathrm{~cm}^{2}$ and $22.8 \mathrm{~cm}^{2}$ ) composed of larger racks, but also of paper notes and notebooks, or physical keyboard.

\footnotetext{
${ }^{1}$ We used a Ward hierarchical clustering to define cut-off sizes.

${ }^{2}$ See /illustrations/TableAndObjectsSize.png for a visualization of the relative size of these objects in the data attached to the article.
}

(4) 3 larger instruments (between $29.6 \mathrm{~cm}^{2}$ and $39 \mathrm{~cm}^{2}$ ). The large objects had a height of 3 to $5 \mathrm{~cm}$.

Among these objects, the hybrid objects, namely racks of tubes with a digital augmentation were the most consistent in size. Given their medium size the hybrid racks occluded on average $5.16 \%$ of the display $(S D=2 \%$ ). Whereas passive objects occluded $3.21 \%$ of the display $(S D=2.4 \%)$

\section{Presence of objects and occlusion}

We observed between 4 and 8 objects on the tabletop at a point in time (avg. $=5.6, S D=1.8$ ). On average, an object was used during one third of a session (same for passive and hybrid, with $S D=14.5 \%$ ).

Based on our measures of the size and presence of objects, we calculated that on average $15.5 \%$ of the screen of the eLabBench was occluded at any point in time $(S D=4.9 \%)$. Passive objects accounted for $12.0 \%$ of the occlusion and hybrid ones for $4.4 \%$ (respectively $S D=6.1 \%$ and $2.6 \%$ ).

\section{Interaction and Object Organization}

In order to analyze how participants organized objects on the tabletop, we computed a set of metrics. These include: the position of objects throughout the sessions, the distance an object was moved, the number of times an object was moved on and off the tabletop.

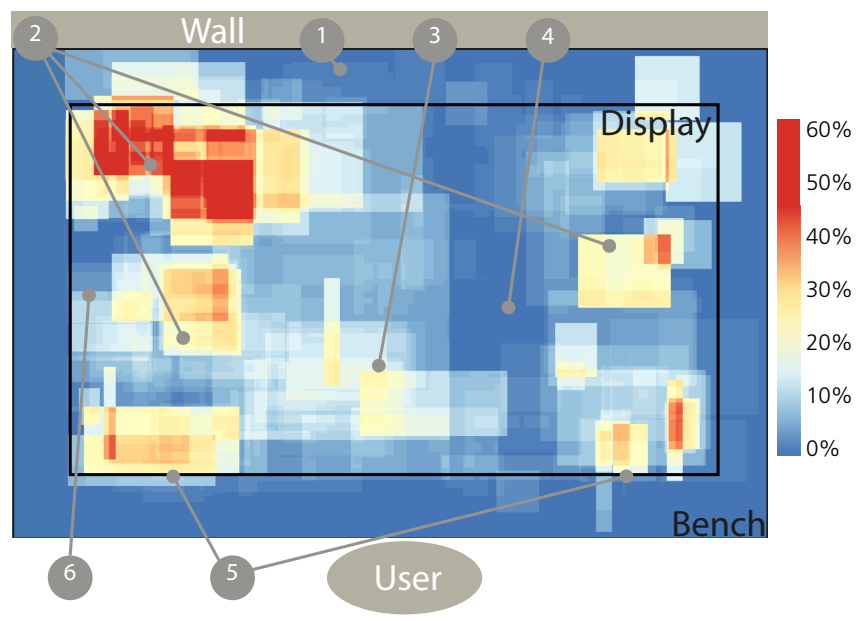

Figure 2. Overview of physical objects' position on the tabletop over the 5 sessions. From blue: display not occluded, to light blue: display occluded $20 \%$ of the time, to yellow $(30 \%)$ to red $(60 \%)$.

\section{Position}

We observed 5 different zones where participants placed objects. The heatmap in fig. 2 illustrates the activity and the position of the zones described below:

1. Long term storage area, where objects are stored from one session to the next, at the top of the bench close to the wall. This row of objects can be seen in fig. 1, but does not appear on the heatmap as we did not annotate these objects which were not used and did not occlude the display.

2. Storage areas, where objects are pushed to and which heavily occlude the display (left side of the bench, and slightly less on the right side) $)^{3}$.

\footnotetext{
${ }^{3}$ See /heatmaps/high-presence.pdf for a heatmap of objects staying on the bench for more than half of the sessions' duration, and
} 
3. The observation area, where objects are placed for short periods of time, to be observed or used.

4. The digital area where digital content is placed, and left free of physical objects. The right-handedness of the participants might account for the area position.

5. Interaction areas where often used objects are stored (e.g. pen, keyboard, mouse, pipettes, tube caps, etc.) This area is mostly occluded by small items (see also fig. 3).

We also noted an area less occluded at the left of the bench (6), which is the location of the main menu of the eLabBench.

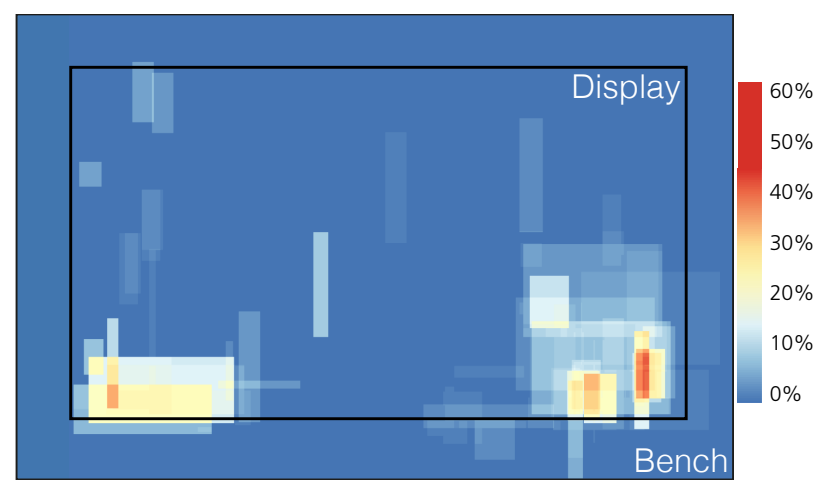

Figure 3. Heatmap of objects participants mostly interacted with.

\section{Mobility}

The majority of the objects brought to the eLabBench were moved around (72\%). The most common action was to drop an item on the surface when performing a task that required both hands. For example, dropping a pen on the bench to pipette something, or dropping a pipette to take some notes. In such cases, objects were placed on the surface for a short period of time, and moved very little. These objects were relatively small and placed in the interaction area (see fig. 3).

A second category of object movement happened when participants brought an object closer to themselves to interact with it, and then moved it back to a zone further away. For example taking a rack of tubes for pipetting, and then moving it back to the storage area once done with the task. In these situations, objects are typically moved less than $60 \mathrm{~cm}$, between the periphery of the bench (zone 2) and the center (zone 3 in fig. 2). Finally, participants moved objects in and out of the tabletop. For example, when getting some samples in a rack from the fridge and putting them back when finished.

\section{Occlusion of digital elements}

The eLabBench did not handle occlusion, which led most participants to carefully place physical and digital objects to minimize their overlap and resulting occlusion. Fig. 4 shows a heatmap of the digital objects occluded by physical ones, most of the display is blue meaning that digital elements were not occluded, or very little.

Nonetheless, given the large number of objects used on the tabletop, some digital widgets and documents were still occluded. The storage zone is where significant occlusion happens, with the display being occluded during $10 \%$ to $23 \%$ of

/heatmaps/static.pdf for objects which did not move throughout the sessions. a session's duration (white/yellow spots in fig. 4). This heavy occlusion zone is surrounded by an area with occasional occlusion (light blue). The Interaction area at the bottom of the screen where participants placed objects which they needed often (keyboard, pipette, pen) is the second area with occlusion. In this area, participants made an effort to limit the number of digital elements in order to avoid occlusion and to avoid triggering false touch events when dropping or grabbing physical items ${ }^{4}$.

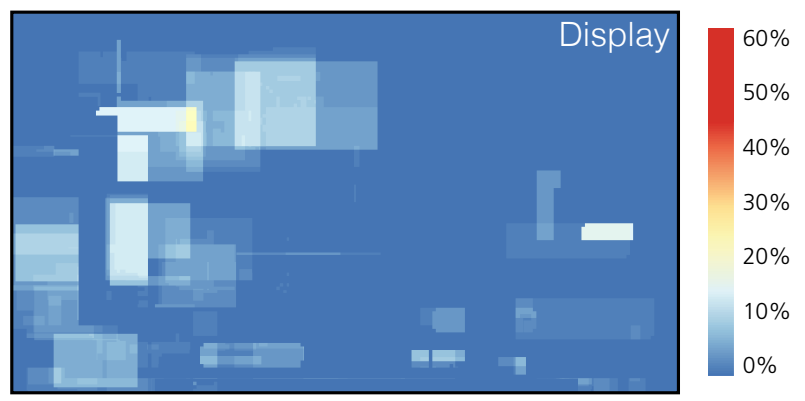

Figure 4. Levels of occlusion of digital objects (documents and widgets) by physical objects (passive and hybrid). Blue: display occluded less than $5 \%$ of the time. Yellow: $10 \%$. Orange $20 \%$.

Throughout the deployment of the eLabBench users did not complain about occlusion issues. One reason could be that we were clear that occlusion would not be handled when introducing the device, but we also raised other limitations which, unlike occlusion, participants kept complaining about (such as limited leg room under the tabletop). When being more specific and asking about the relationship between physical objects and digital ones, participants raised the recognition of objects as touch or pen events as the main issue.

\section{Limitations}

The data discussed here comes from the deployment of a specific tabletop application designed for supporting single-user laboratory work, where physical objects are numerous and necessary. While we believe that the eLabBench would be used similarly in other laboratory work contexts (engineering, chemistry, medicine...), studies are needed to extend our results to other situations. Our analysis is however based on field data, which proves to be less stereotypical in the variety of objects used, but also their positioning. Whether in office or entertainment situations, chances are that tabletop displays will not be occluded only by coffee mugs, laptops or A4 paper documents, usually used in research related to occlusion.

Finally if our system had handled occlusion we might have observed different behaviors. In such a chicken and egg situation, we see the value of our data and analysis in offering real world use cases of objects and occlusion management to guide interaction designers. To this end, we attached our data to the article for testing or bench-marking future occlusion management techniques.

\section{DISCUSSION AND IMPLICATIONS FOR DESIGN}

An important lesson from the eLabBench deployment was that the tabletop display played an ambient role [10].

\footnotetext{
${ }^{4}$ See /heatmaps/digital-map.pdf for a heatmap of digital resources positions.
} 
Participants primarily focused on running their laboratory work, fetching resources, preparing material, pipetting, thus mainly interacting with physical material on top of the surface. They only sporadically checked digital protocols or took digital notes. In this context, occlusion management techniques should limit distractions by using little animations and visual changes as new objects are put on the table.

A new direction to limit distraction and changes whenever objects are placed on the tabletop would be to explore preemptive actions (see [4] for such an instance), rather than focusing solely on reactive techniques (used in $[3,5,6,7]$ ). Occlusion could be prevented by automatically positioning digital objects in locations where they are unlikely to be occluded, which we observed participants manually do.

Indeed, our observations suggest that participants organize digital and physical objects in a meaningful way, by e.g. pushing less-used objects away and keeping often-used objects in close proximity. This is quite natural given the large degree of expertise people have managing objects. However when handling digital elements, it can sometimes be difficult to move menus or palettes which are located in fixed position, or to group, split or organize sets of items together. Tabletops are well suited to support such interactions which could be integrated into occlusion management techniques, for instance by allowing users to move a set of digital objects as a whole, before putting a physical object on the tabletop.

Many of the objects on the elabBench were 3 to $5 \mathrm{~cm}$ high, which means that digital elements were occluded even though they had no objects right on top. In such situations, a simple user model or more advanced user tracking could help determine which parts of the screen are actually occluded as suggested in [6]. Moreover, the height of objects does not only impede vision but also interaction, making digital elements located behind physical ones hard to reach, which should be taken care of in occlusion management techniques.

Participants tended to push objects toward the periphery of the screen in order to keep the central part visible. Therefore, designers should beware of placing significant UI elements close to the edges of the display. In the case of the eLabBench, the docked menu attached to the left side of the display was a bad design choice; contextual menus or palettes would have been better.

Finally, designers should fine tune the occlusion management techniques they design to the type and position of objects. For instance, participants often picked and dropped objects in the interaction zone. In this area, objects were relatively small and hence did not impede participants from viewing the display, but picking them would easily trigger touch events (the eLabBench relied on an interactive pen so this was not observed). If the borders around objects were to become interactive, unintended touches could happen when grabbing or dragging physical objects.

\section{CONCLUSION}

Based on the field deployment of an interactive tabletop system for laboratory work, we investigated how users managed physical and digital objects and the resulting occlusion. We first described and classified the quantity, size and presence of objets used. We then analyzed object management, looking at how participants placed and interacted with objects in different areas of the tabletop. Our results show five areas on the tabletop: Long term storage, session storage, observation area, interactive area and a digital area. Finally, we described how participants moved objects on the surface of the tabletop and handled occlusion of digital objects by physical ones.

From this, we derived design implications, particularly for handling occlusion. We emphasized the risks of animations and highly reactive occlusion management techniques in distracting users from their primary tasks. We thus propose to consider occlusion not as a static problem of "one physical object hiding digital elements at a point in time", to which the display should react, but rather as a situation which should be prevented. This implies for instance, pre-positioning digital elements in locations where they are not likely to be occluded or acknowledging that some objects are deliberately put in occluding positions because users want to interact with them conveniently and should not necessarily trigger an immediate occlusion management response.

\section{ACKNOWLEDGMENTS}

We thank Ebbe S. Andersen and the deployment participants for making this research possible. This research was funded by the Danish Agency for Science, Technology, and Innovation under the project "PC Mini-Grids for Prediction of Viral RNA Structure and Evolution", \#09-061856.

\section{REFERENCES}

1. Aubert, O., and Prié, Y. Advene: active reading through hypervideo. In Proceedings of HYPERTEXT'05, ACM (2005), 235-244.

2. Brandl, P., Leitner, J., Seifried, T., Haller, M., Doray, B., and To, P. Occlusion-aware menu design for digital tabletops. In Proceedings of CHI EA'09, ACM (2009), 3223-3228.

3. Cotting, D., and Gross, M. Interactive environment-aware display bubbles. In Proceedings of UIST'06, ACM (2006), 245-254.

4. Freeman, E., and Brewster, S. Messy tabletops: clearing up the occlusion problem. In Proceedings of CHI EA '13, ACM (2013), 1515-1520.

5. Furumi, G., Sakamoto, D., and Igarashi, T. Snaprail: a tabletop user interface widget for addressing occlusion by physical objects. In Proceedings of ITS '12, ACM (2012), 193-196.

6. Javed, W., Kim, K., Ghani, S., and Elmqvist, N. Evaluating physical/virtual occlusion management techniques for horizontal displays. In Proceedings of INTERACT'11, Springer-Verlag (2011), 391-408.

7. Khalilbeigi, M., Schmittat, P., Mühlhäuser, M., and Steimle, J. Occlusion-aware interaction techniques for tabletop systems. In Proceedings of CHI EA'12, ACM (2012), 2531-2536.

8. Scott, S. D., Carpendale, M. S. T., and Inkpen, K. M. Territoriality in collaborative tabletop workspaces. In Proceedings of $\mathrm{CSCW}^{\prime} 04, \mathrm{ACM}$ (2004), 294-303.

9. Steimle, J., Khalilbeigi, M., Mühlhäuser, M., and Hollan, J. D. Physical and digital media usage patterns on interactive tabletop surfaces. In Proceedings of ITS'10, ACM (2010), 167-176.

10. Tabard, A., Hincapié Ramos, J. D., and Bardram, J. The elabbench in the wild: supporting exploration in a molecular biology lab. In Proceedings of CHI'12, ACM (2012), 3051-3060.

11. Tabard, A., Hincapié-Ramos, J.-D., Esbensen, M., and Bardram, J. E. The elabbench: an interactive tabletop system for the biology laboratory. In Proceedings of ITS'11, ACM (2011), 202-211.

12. Toney, A., and Thomas, B. H. Considering reach in tangible and table top design. In Proceedings of TableTop'06., IEEE (2006), 2-10.

13. Vogel, D., and Casiez, G. Hand occlusion on a multi-touch tabletop. In Proceedings of CHI'12, ACM (2012), 2307-2316. 